\title{
2 und 3.
}

Aus der chirurgischen Abtheilung der Magdeburger Krankenanstalt Sudenburg.

2. Aneurysma spurium der Femoralis, combinirt mit Varix aneurysmaticus.

Von

Oberarzt Dr. Habs.

(Mit 1 Abbildung.)

Es handelt sich bei der folgenden Mittheilung einerseits um ein Aneurysma spurium der Arteria femoralis und andererseits zugleich um ein Varix aneurysmaticus der Vena femoralis, beide entstanden durch einen Messerstich, der Arterie und Vene zugleich perforirte.

Wir haben also erstens ein Aneurysma spurium, ein solches stellt bekanntlich einen blutgefillten Sack dar, welcher direct mit dem Arterienlumen communicirt, und dessen Wand durch Blutgerinnsel und durch das plastisch infiltrirte Gewebe der Ungebung gebildet wird, im Gegensatz zum Aneurysma verum, welches ja eine sackartige Erweiterung des Arterienrohres darstellt, und dessen Wandung von sämmtlichen Elementen der Gefässwand gebildet wird.

Des Weiteren haben wir, wie gesagt, an unserem Präparat zugleich einen Varix aneurysmaticus. Ein soleher kommt zu Stande dadurch, dass eine Oeffnung in der Arterie mit einer correspondirenden Oeffnung der Vene erwächst, so dass sich zwischen Arterie und Vene gewissermaassen eine Lippenfistel bildet. Hat sich dies ereignet, so strömt das arterielle Blut durch die Fistel in die Vene, wie in einen Arterienast hinein, begegnet dort dem Blutstrome der Vene und führt durch Steigerung des intravenösen Druckes zu einer Ausweitung der Vene. Diese Ausdehnung der Vene nennen wir einen Varix aneurysmaticus.

Krankengeschichte: Paul A., 19 Jahre alt, Kaufmann, stach sich am 19. Juni 1896 versehentlich in den linken Oberschenkel, hatte eine schwere, fast letale Blutung aus der Wunde, welche jedoch auf Compressivverband stand. Ueber den Charakter der Blutung (ob arteriell oder venös) war nichts zu eruiren. Die Stichwunde heilte prima. 4 Wochen nach der Verletzung begann sich das Aneurysma auszubilden und nahm ständig an Grösse zu.

Am 2. November 1896 wurde Patient in die Krankenanstalt Sudenburg mit folgendem Befund anfgenommen: Apfelgrosser Tumor am linken Oberschenkel, welcher synchron der R. femoralis pulsirt, und über welchem ein deutliches Schwirren fühlbar ist, das sich distal, $d$. h. unterhalb des Tumors noch weithin verfolgen lässt. Die Auscultation ergiebt ein lautes, brausendes Geräusch über dem Tumor und auch distal desselben.

Beim Stehen des Patienten ist der Tumor weit mehr gespannt, als beim Liegen. Der Tumor lässt sich durch Compression verkleinern, aber nicht ganz wegdrücken. Von den umgebenden Geweben lässt sich der Tumor nicht abgrenzen, sondern ist überall mit der Musculatur diffus verwachsen. Die Venen des linken Unterschenkels sind stärker geschwellt als die des rechten, jedoch ohne Undulation. Kein Oedema pedis. Puls an der Dorsalis pedis und Tibialis postica beiderseits nicht zu fühlen. 
Die Diagnose auf Aneurysma war klar; die auf Varix aneurysmatica habe ich aber auch ante operationem für wahrscheinlich erklärt, einmal, weil die subcutanen Venen am Unterschenkel der kranken Seite stärker geschwellt waren (wenn auch ohne Cndulation) und zweitens, weil auch unterhalb des Tumors ein starkes Schwirren fühlbar, ein lautes Brausen hörbar war.

Wegen der Grössenzunahme der Aneurysma entschloss ich mich, bei der Unzulänglichkeit aller sonstigen Maassuahmen zur Operation, und zwar zur totalen Entfernung des Sackes. -

Bei der Operation (3. November 1896) lagerte ich den Kranken wie zur Femoralisunterbindung auf den Rücken, den Schenkel im Hüftgelenk fast rechtwinklig abducirt, leicht flectirt und maximal nach aussen rotirt. - An der Aussenseite des Gliedes stehend futhrte ich den Hautschnitt sofort in einer Ausdehnung von etwa $20 \mathrm{Cm}$. von unten nach oben, parallel dem Verlaufe des Sartorius, über die Geschwulst hinweg. Künstliche Blutleere wandte ich nicht an, da durch die in nächster Nähe des Operationsterrains anzulegende Gummibinde die Asepsis gefährdet erschien, und da ich mich an dem pulsirenden Tumor besser orientiren $z u$ können glaubte.

Zunächst legte icb dann, um jederzeit einer grösseren Blutung Herr werden zu können, die Arteria femoralis im oberen Wundwinkel, etwa $7 \mathrm{Cm}$. unterhalb des Lig. P'ouparti frei und führte provisorisch einen Seidenfaden um dieselbe, der aber nicht geknüpft wurde.

Diese provisorische Ligatur legte ich, wie gesagt, $7 \mathrm{Cm}$. unterhalb des Poupart'schen Bandes an, nicht an der weit bequemeren klassischen Stelle dicht unterhalb desselben; ich that dies in der Erwägnng, dass die Profunda $31 / 2$ und die beiden Circumflexae $5 \mathrm{~cm}$. unterhalb des Bandes entspringen. Es war also, falls diese Ligatur hătte geknüpft werden müssen, ein genutgender Collateralkreislauf gewährleistet.

Nunmehr wurde der ganze Sack, welcher diffus mit der umgebenden Mnsculatur verwachsen war, mulsselig mit dem Messer ringsum ausgelöst, wobei vorn ganze Partien des fächerförmig entfalteten Sartorius, hinten die Fascie der Adductoren mit weggenommen werden musste.

Schliesslich wurden die grossen Gefässe am unteren Pol des Aneurysma im Hunter'schen Kanale freigelegt, und es zeigte sich hierbei, dass, meiner Vermuthungsdiagnose entsprechend, die sebr stark ausgedehnte Vene deutlich pulsirte, fast so stark wie die Arterie.

Da mir wan diese Dilatation und Pulsation ein Beweis war, dass eine Communication zwischen Arterie und Vene bestand, so entschloss ich mich zur Resection beider Gefässe.

Um aber nicht zu.viel zu reseciren, d. b. um möglichst wenig Nebenäste zu opfern, musste ich wich über die Lage der Oeffnung in der Arterie orientiren. Unter Digitalcompression des Arterienstammes eröffinete ich den Aneurysmasack mittelst eines Längsschnittes und entfernte aus demselben die Coagula (ein hühnereigrosses und mehrere wandständige kleine). Sofort sprang, bei Nachlass der Compression, aus der Arterienöffnung Blut im Strahle hervor. Dicht oberhalb und unterhalb dieser stelle unterband ich Arterie und Vene, jede für sich mit doppelter Ligatur, und durchschnitt sie, wodurch der Aneurysmasack vollkommen entfernt wurde. 
Der provisorisch um die Arterie an höherer Stelle gelegte Faden wurde wieder entfernt. Der Blutverlust bei der Operation war ein minimaler. Die mächtige Wunde wurde durch eine Reihe von Seidenknopfnähten geschlossen, und ist dieselbe prima und ohne Fieber geheilt.

Die Circulation im Fusse war gleich nach der Operation gut und hat auch in der Folge keinerlei Störungen erlitten. Um Wiederherstellung des Kreislaufes waren wir auch bei der Operation nicht so sehr besorgt, da wir ja unterhalb des Abganges der Profunda und der Circumflexae operirt hatten, und da ja diese Arterien von oben her genügend Blut in die Muskeläste der Poplitaea und durch dieselbe rückläufig in den Stamm der Poplitaea führen können. - Als interessantes, wohl hierher gehöriges Symptom möchte ich hervorheben, dass die grosse Hautnarbe in ihrer unteren Hälfte kelö̈dartig entartet iat, ein Vorkommniss, dessen Grund vielleicht in geringen Circulationsstörungen zu finden sein dürfte.

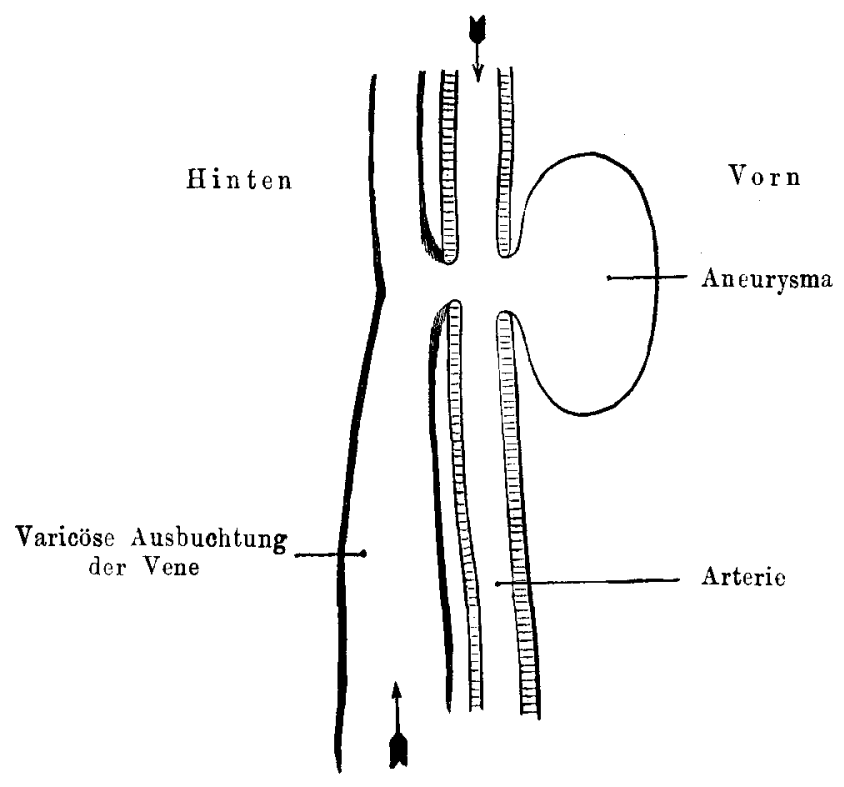

An dem Präparat (vergl. nebenstehende schematische Zeichnung) ist sehr deutlich zu erkennen, dass der Stich durch Arterie und Vene hindurchgegangen ist. Die Oeffnung in der Arterie communicirt mit dem Aneurysma, welches vor der Arterie gelegen ist. Die hintere Arterienöffnung ist mit der vorderen Venenöffnung lippenartig verwachsen. Der Stich in der hinteren Venenwand ist fest vernarbt, man sieht aber an dieser Stelle die Narbe noch dentlich als kleine trichterförmige Einsenkung. Die Vene zeigt distal von der Verletzungsstelle eine um das Doppelte weiteres Lumen als proximal.

Das Präparat zeigt uns also die seltene Combination eines Aneurysma spurium arteriae femoralis mit einem Varix aneurysmaticus. 